\title{
Langmuir
}

pubs.acs.org/Langmuir

(C) XXXX American Chemical Society

\section{Three-Dimensional Structure and Growth of Myelins}

\author{
Louisa Reissig, ${ }^{\dagger \dagger, \S}$ David J. Fairhurst, ${ }^{\dagger, \|}$ Jacques Leng, ${ }^{\dagger \dagger}, \perp$ Michael E. Cates,${ }^{\dagger}$ Andrew R. Mount,,$* \dot{t}$ \\ and Stefan U. Egelhaaf*,;,\# \\ ${ }^{\dagger}$ SUPA, School of Physics and Astronomy, The University of Edinburgh, Edinburgh EH9 3JZ, United Kingdom, \\ ${ }^{*}$ EaStCHEM, School of Chemistry, The University of Edinburgh, Edinburgh EH9 3JJ, United Kingdom, \\ ${ }^{\S}$ Department of Biophysics, Institute of Experimental Physics, Warsaw University, 02-089 Warsaw, Poland, \\ "Department of Physics, Nottingham Trent University, Nottingham NG11 8NS, United Kingdom, \\ ${ }^{\perp}$ Laboratoire du Futur, Université Bordeaux-1, 33608 Pessac cedex, France, and ${ }^{\#}$ Condensed Matter Physics \\ Laboratory, Heinrich-Heine-University, 40225 Düsseldorf, Germany
}

Received July 7, 2010. Revised Manuscript Received August 15, 2010

\begin{abstract}
After contact with water, surfactant lamellar phases $\left(\mathrm{L}_{\alpha}\right)$ can show spectacular interface instabilities: multibilayer tubules, so-called myelins, grow from the $\mathrm{L}_{\alpha}$ /water interface into the water. We have studied the shape, size, and growth of myelins in aqueous solutions of the nonionic surfactant $\mathrm{C}_{12} \mathrm{E}_{3}$ (triethylene glycol monododecyl ether) during dissolution. We used a combination of different imaging techniques: optical microscopy providing 2-D projections of the sample and confocal microscopy offering a complete 3-D reconstruction. These techniques provide quantitative information on the shape and growth of myelins, such as their width, length, and depth profile as a function of time. The growth rate of myelins, characterized by a swelling or diffusion coefficient, was found to increase with surfactant mass fraction and, seemingly, with sample thickness. We demonstrate that myelin creaming due to buoyancy can explain the apparent dependence on sample thickness. Our experiments furthermore suggest that myelin growth is controlled by an interplay between the water mobility in the lamellar phase and the osmotic pressure difference between the lamellar phase and the contacting water.
\end{abstract}

\section{Introduction}

Adding water to surfactant phases, such as soap, is an apparently mundane, everyday "experiment", which can nevertheless show many surprising and interesting features. We are particularly interested in the spontaneous formation of nonequilibrium cylindrical structures, so-called myelins. These interface instabilities can form during the dissolution and swelling of surfactant lamellar phases. Despite an increasing interest in these structures, ${ }^{1-24}$

*Corresponding authors. E-mail: A.Mount@ed.ac.uk (A.R.M.); Stefan.Egelhaaf@uni-duesseldorf.de (S.U.E.).

(1) Buchanan, M.; Egelhaaf, S. U.; Cates, M. E. Langmuir 2000, 16, 3718-3726.

(2) Buchanan, M. Dynamics of Interfaces in Surfactant Lamellar Phases.

$\mathrm{PhD}$ Thesis, The University of Edinburgh, 1999.

(3) Kennedy, A. P.; Sutcliffe, J.; Cheng, J.-X. Langmuir 2005, 21, 6478-6486.

(4) Sakurai, I.; Suzuki, T.; Sakurai, S. Mol. Cryst. Liq. Cryst. 1990, 180B, 305-311.

(5) Sakurai, I.; Suzuki, T.; Sakurai, S. Biochim. Biophys. Acta 1989, 985, 101-105.

(6) Stoeckenius, W. J. Biophys. Biochem. Cytol. 1959, 5, 491-500.

(7) Haran, M.; Chowdhury, A.; Manohar, C.; Bellare, J. Colloids Surf., A 2002, 205, 21-30.

(8) Neuzil, E.; Fourche, J.; Jensen, R.; Jensen, H.; Morin, G. Biochim. Biophys. Acta 1981, 641, 11-19.

(9) Degkwitz, R. Protoplasma 1938, 31, 180-183.

(10) Sandermann, H.; Falk, H.; Schumacher, G. Anal. Biochem. 1977, 82, $583-585$.

(11) Vogel-Weill, C.; Gruger, A.; Neuzil, E. C. R. Acad. Sci. Paris 1991, 312, 1077-1082.

(12) Gruger, A.; Vogel-Weill, C. Mol. Cryst. Liq. Cryst. 1994, 238, 227-239

(13) Arunagirinathan, M. A.; Roy, M.; Dua, A. K.; Manohar, C.; Bellare, J. R. Langmuir 2004, 20, 4816-4822.

(14) Zou, L.-N. Phys. Rev. E 2009, 79, 061502

(15) Zou, L.-N.; Nagel, S. R. Phys. Rev. Lett. 2006, 96, 138301

(16) Chen, B.-H.; Miller, C. A.; Walsh, J. M.; Warren, P. B.; Ruddock, J. N.; Garrett, P. R.; Argoul, F.; Leger, C. Langmuir 2000, 16, 5276-5283.

(17) Nageotte, J. Morphologie des gels lipoides, myéline, cristaux liquides, vacuoles; Hermann \& cie: Paris, 1936.

(18) Buchanan, M.; Egelhaaf, S. U.; Cates, M. E. Colloids Surf., A 2001, 183, 293-302.

(19) Huang, J.-R.; Zou, L.-N.; Witten, T. A. Eur. Phys. J. E 2005, 18, 279-285. there is currently no accepted explanation for the formation of myelins.

Myelins can form when water is added to a lamellar phase $\left(\mathrm{L}_{\alpha}\right)$ which has a large coexistence region of the lamellar phase with water (W). ${ }^{1,2}$ Myelins are multibilayer tubes. ${ }^{3-6}$ The bilayer spacing within the myelins corresponds to the maximum stable swelling of the lamellar phase, which occurs at the boundary with the coexistence region. ${ }^{3,4}$ Many individual tubes are closely packed resulting in a packing fraction of the tubes of $\phi_{\mathrm{cyl}}=$ $\sqrt{ } 3 \pi / 6 \approx 90.7 \%$. ${ }^{1}$ For myelins to form, the bilayers need to be fluid and of low permeability. ${ }^{7-13}$ The addition of molecules which increase the permeability of water ${ }^{7}$ or hinder molecular movement within the bilayers ${ }^{8,9}$ suppresses myelin formation as does solidifying the bilayers, e.g., by lowering the temperature. ${ }^{10,25}$

After contact with water, water enters the lamellar phase via the myelin roots (the $\mathrm{L}_{\alpha}$ /myelin interface) and causes the lamellar phase to swell. ${ }^{1,2}$ This pushes the myelins further into the surrounding water. Myelins have been observed to grow, i.e., increase in length, according to a diffusion-like, square-root dependence on time. ${ }^{1,2,14-16}$ The growth rate can be characterized by a swelling or diffusion coefficient, which was found to depend on the initial surfactant concentration. These experiments suggest that myelin growth is driven by the osmotic pressure difference between the lamellar phase and the contacting water and limited by the water mobility in the lamellar phase. Further models to

(20) Sakurai, I.; Kawamura, Y. Biochim. Biophys. Acta 1984, 777, 347-351.

(21) Sakurai, I. Biochim. Biophys. Acta 1985, 815, 149-152.

(22) Mishima, K.; Yoshiyama, K. Biochim. Biophys. Acta 1987, 904, 149-153.

(23) Dave, H.; Surve, M.; Manohar, C.; Bellare, J. J. Colloid Interface Sci. 2003, $264,76-81$.

(24) Taribagil, R.; Arunagiritnathan, M. A.; Manohar, C.; Bellare, J. J. Colloid Interface Sci. 2005, 289, 242-248.

(25) Fairhurst, D. J.; Baker, M. E.; Shaw, N.; Egelhaaf, S. U. Appl. Phys. Lett. 2008, 92,174105 . 
describe swelling of lamellar phase and myelin growth have been proposed. $^{20-25}$

Here we investigate myelin formation in aqueous solutions of the nonionic surfactant $\mathrm{C}_{12} \mathrm{E}_{3}$ (triethylene glycol monododecyl ether, $\left.\mathrm{CH}_{3}\left(\mathrm{CH}_{2}\right)_{11}\left(\mathrm{OCH}_{2} \mathrm{CH}_{2}\right)_{3} \mathrm{OH}\right)$. The equilibrium phase diagram of aqueous $\mathrm{C}_{12} \mathrm{E}_{3}$ solutions is well established. ${ }^{26-30}$ At room temperature $\left(T \approx 20^{\circ} \mathrm{C}\right)$ a pure lamellar phase $\left(\mathrm{L}_{\alpha}\right)$ is observed for surfactant mass fractions, $\phi$, in the range $37 \%<\phi<81 \%$. For lower surfactant concentrations a coexistence region of this phase with water $\left(\mathrm{L}_{\alpha}+\mathrm{W}\right)$ is found, and at higher surfactant concentrations there is a coexistence region with surfactant phase $\left(\mathrm{L}_{\alpha}+\right.$ $\left.\mathrm{L}_{2}\right)$. In the most concentrated $\mathrm{C}_{12} \mathrm{E}_{3}$ solutions $(\phi>84 \%)$ inverted micelles $\left(\mathrm{L}_{2}\right)$ are present. The molecular structure of $\mathrm{C}_{12} \mathrm{E}_{3}$ allows the ethylene oxide head groups to form hydrogen bonds with water as well as between each other. ${ }^{29-32}$ This network of hydrogen bonds is similar to that in poly(ethylene oxide); with increasing water concentration helical conformations are found which trap an average of 2.9 water molecules per ethylene oxide unit. $^{33,34}$ This structural motif can be found in all liquid phases as well as in the myelins. ${ }^{3,30,32}$ The hydrocarbon chains are, especially in $\mathrm{L}_{\alpha}$, predominantly in an all-trans configuration, except close to the headgroup. This renders the interface flexible with respect to the conformation and orientation of the chains. ${ }^{35-38}$ Studies on monolayers have shown that the interfacial area per molecule is about $0.36 \mathrm{~nm}^{2}$ and the thickness of the monolayer is $2.3 \mathrm{~nm}$, with the chain and headgroup contributing 1.25 and $1.05 \mathrm{~nm}$, respectively. ${ }^{39-41}$

The formation of myelins in $\mathrm{C}_{12} \mathrm{E}_{3}$ samples has been studied previously and their growth found to be relatively slow. ${ }^{1-3,16,26}$ They form at the $\mathrm{L}_{\alpha} / \mathrm{W}$ interface and are long-lived metastable structures with their bilayer spacing equal to the maximally swollen state. ${ }^{3}$ Here we use a combination of imaging techniques, conventional and confocal microscopy, to investigate the shape, size, and growth of myelins for different initial surfactant mass fractions, $\phi$, and sample thicknesses, $b$. Our results indicate that both myelin formation and water influx occur homogeneously over the whole interface. They furthermore suggest that myelin growth is controlled by the interplay between the osmotic pressure difference between the lamellar phase and the contacting water, which represents the driving force, and the water mobility in the lamellar phase.

(26) Laughlin, R. G. In Micelles, Microemulsions and Monolayers; Shah, D. O., Ed.; Marcel Dekker: New York, 1998; pp 73-100.

(27) Laughlin, R. G.; Lynch, M. L.; Marcott, C.; Munyon, R. L.; Marrer, A. M Kochvar, K. A. J. Phys. Chem. B 2000, 104, 7354-7362.

(28) Hung, K.-C.; Chen, B.-H. AIChE J. 2007, 53, 1450-1459.

(29) Mitchell, D. J.; Tiddy, G. J. T.; Waring, L.; Bostock, T.; McDonald, M. P. J. Chem. Soc., Faraday Trans. 1 1983, 79, 975-1000.

(30) Oswald, P.; Pieranski, P. Nematic and Cholesteric Liquid Crystals: Concepts and Physical Properties Illustrated by Experiments; Taylor \& Francis: Boca Raton, FL, 2005.

(31) Ohno, K.; Takao, H.; Masuda, T.; Katxumoto, Y. Chem. Lett. 2005, 34 , 250-251

(32) Ohno, K.; Takao, H.; Katsumoto, Y. Spectrochim. Acta, Part A 2006, 63, $690-693$.

(33) Tasaki, K. J. Am. Chem. Soc. 1996, 118, 8459-8469.

(34) Begum, R.; Matsuura, H. J. Chem. Soc., Faraday Trans. 1997, 93, 3839 3848 .

(35) Tonegawa, A.; Ohno, K.; Matsuura, H.; Yamada, K.; Okuda, T. Chem. Lett. 2000, 29, 324-325.

(36) Masatoki, S.; Ohno, K.; Yoshida, H.; Matsuura, H. Chem. Lett. 1996, 25, $149-150$

(37) Masatoki, S.; Ohno, K.; Yoshida, H.; Matsuura, H. J. Phys. Chem. 1996 100, 8487-8498.

(38) Matsuura, H. Prog. Colloid Polym. Sci. 1997, 106, 42-48.

(39) Bell, G. R.; Bain, C. D.; Ward, R. N. J. Chem. Soc., Faraday Trans. 1996, $92,515-523$.

(40) Lu, J. R.; Hromadova, M.; Thomas, R. K.; Penfold, J. Langmuir 1993, 9, 2417-2425.

(41) Lu, J. R.; Lee, E. M.; Thomas, R. K.; Penfold, J.; Flitsch, S. L. Langmuir 1993, 9, 1352-1360.

\section{Materials and Methods}

2.1. Materials and Sample Preparation. All chemicals were used as received. $\mathrm{C}_{12} \mathrm{E}_{3}$ (Nikkon Chemicals, purity $99.9 \%$ ) was stored under nitrogen at $5^{\circ} \mathrm{C}$. By adding Millipore water, aqueous solutions of $\mathrm{C}_{12} \mathrm{E}_{3}$ were prepared with different $\phi$. The samples were homogenized (either by shaking using a mechanical shaker or by rotating the sample once per second) for several hours. They were then left at room temperature $\left(T=20 \pm 2{ }^{\circ} \mathrm{C}\right)$ for several days to equilibrate. The samples were then observed visually by eye through crossed polarizers. The lamellar phase shows characteristic birefringence and, at high $\phi$, significant turbidity. We can thus visually determine phase separation into a turbid, birefringent $\mathrm{L}_{\alpha}$ phase and a clear $\mathrm{L}_{2}$ phase as well as into a birefringent $\mathrm{L}_{\alpha}$ phase and water. For confocal microscopy, samples at $\phi=65 \%$ were prepared with trace amounts of the dye Rhodamine B (Fluka, standard Fluka grade). This trace amount of dye did not cause an observable change in the phase behavior.

To investigate structural changes during dissolution, we performed experiments similar to penetration scans, a method typically used to study surfactant phase behavior. ${ }^{42}$ A droplet of surfactant solution with surfactant mass fraction $\phi$ was sandwiched between two glass plates, which were separated by a spacer of thickness $b$. During sample preparation, the surfactant solution was squeezed between the two glass plates and thus sheared. This procedure results in clean, vertical interfaces between the droplet and air (and later water) and significantly improved reproducibility. The experiment was started by placing Millipore water between the glass plates, which was brought into contact with the droplet through capillary forces. Typically five individual measurements for each condition have been performed and averaged. All experiments were performed at room temperature $\left(T=20 \pm 2^{\circ} \mathrm{C}\right)$.

2.2. Microscopy. An Olympus BX50 microscope with a $10 \times$ phase contrast objective was used to image the samples. The images were manually analyzed to determine myelin width; typically about 100 myelins were measured for each experimental condition.

2.3. Direct Observation. A home-built imaging device with a magnification of between 0.5 and $2.5^{43,44}$ was used to monitor an entire droplet with a diameter of typically 5-10 $\mathrm{mm}$. This apparatus allowed us to (just) resolve individual myelins and at the same time observe thousands of myelins in the field of view. This resulted in significantly improved statistics.

The sample was illuminated with parallel white light and imaged onto a CCD chip using two identical achromatic doublets (focal length $f=200 \mathrm{~mm}$ ). Images were recorded with a CCD camera at appropriate intervals for several hours. They were analyzed using Image Pro Plus (MediaCybernetics) to locate the outer edge of the droplet and to determine the projected area $A(t)$ of the droplet (including the myelins), from which the mean radius $R(t)=(A(t) / \pi)^{1 / 2}$ was calculated as a function of time $t$ since contact. The mean distance between the myelin tips, i.e. $R(t)$, and the initial position of the interface, i.e. $R\left(t_{0}\right)=R_{0}$, defines the average myelin length $L(t)=R(t)-R_{0}$, as water contacted the droplet at $t_{0}=0 \mathrm{~s}$.

2.4. Confocal Microscopy. A BioRad Radiance 2100 confocal scanning microscope connected to an inverted Nikon Eclipse TE300 microscope with a $10 \times$ objective was used to obtain 3-D images of the samples. The excitation wavelength was $514 \mathrm{~nm}$. To reduce the effect of a finite scan speed on the obtained $z$-profiles, the samples were scanned in both directions, up and down, with a depth resolution of $\Delta z=5 \mu \mathrm{m}$ and the results averaged. Following this procedure enabled a $z$-profile to be determined in about $4 \mathrm{~min}$.

(42) Laughlin, R. G. The Aqueous Phase Behaviour of Surfactants; Academic Press: London, 1996.

(43) Starrs, L. Collapse of Transient Gels in Colloid-Polymer Mixtures. $\mathrm{PhD}$ Thesis, The University of Edinburgh, 1999.

(44) Buchanan, M.; Starrs, L.; Egelhaaf, S. U.; Cates, M. E. Phys. Rev. E 2000, $62,6895-6905$. 


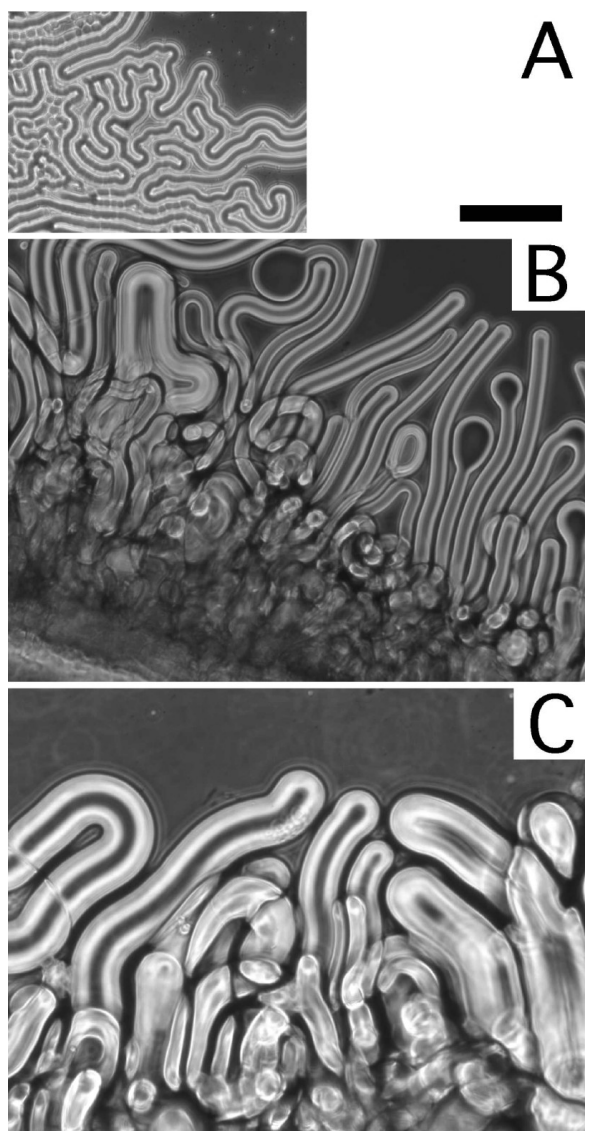

Figure 1. Myelins as observed by optical microscopy. The sample thickness $b$ was (A) $13 \mu \mathrm{m}$, (B) $200 \mu \mathrm{m}$, and (C) $600 \mu \mathrm{m}$, and the surfactant mass fraction $\phi$ was (A, C) $65 \%$ and (B) $60 \%$. The scale bar corresponds to $200 \mu \mathrm{m}$.

\section{Results and Discussion}

3.1. Myelin Shape and Width. The formation and growth of myelins were followed using optical microscopy (Figure 1), which allows us to resolve details of individual myelins. Upon contact with water, the myelins start to form after a delay time $t^{\prime}$ of a few seconds (section 3.2). Myelins grow from the interface of the droplet into the surrounding water. In the case of droplets containing $\mathrm{L}_{2}$ phase, the initial $\mathrm{L}_{2}$ phase changes, after contact with water, rapidly into an $\mathrm{L}_{\alpha}$ phase from which myelins start to grow. The myelins are typically stable for hours. During this time, they grow in length while their width remains constant with time and position along the myelins. However, individual myelins have somewhat different lengths and widths, and some are also curved or branched.

The distribution of the (projected) myelin width $N(d)$ was obtained as a function of the initial surfactant mass fraction $\phi$ and sample thickness $b$. The width $d$ of the myelins was measured manually, typically for about 100 myelins for each condition (Figure 2). The resulting distribution $N(d)$ was modeled by a Schultz distribution

$$
N(d)=\frac{d^{z}}{z !}\left(\frac{z+1}{\langle d\rangle}\right)^{z+1} \exp \left[-\frac{d}{\langle d\rangle}(z+1)\right]
$$

with the average myelin width $\langle d\rangle$ and polydispersity $\sigma=(1 /(z+1))^{1 / 2}$. This distribution describes the data well, with the exception of some outliers at large $d$ and correspondingly small $N(d)$, which could be due to the limited statistics and quantized, integer occurrence.

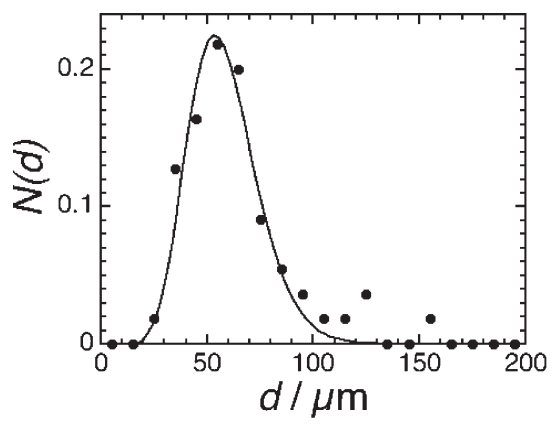

Figure 2. Distribution of the (projected) myelin width $N(d)$ based on the measurement of about 60 individual myelins in a sample with initial surfactant mass fraction $\phi=75 \%$ and sample thickness $b=200 \mu \mathrm{m}$. The line represents a fit by a Schultz distribution with average myelin width $\langle d\rangle=58.3 \mu \mathrm{m}$ and polydispersity $\sigma=0.28$.

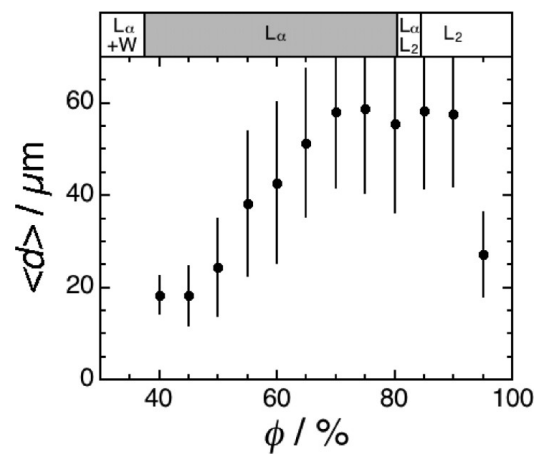

Figure 3. Average myelin width $\langle d\rangle$ as a function of initial surfactant mass fraction $\phi$ for a sample thickness $b=200 \mu \mathrm{m}$. The bars reflect the absolute polydispersity $\sigma\langle d\rangle$. A schematic phase diagram for the corresponding $\phi$ and temperature is shown above.

The average myelin width $\langle d\rangle$ depends on the initial surfactant mass fraction $\phi$ and sample thickness $b$. The dependence of the average width $\langle d\rangle$ on $\phi$ was investigated for a constant sample thickness $b=200 \mu \mathrm{m}$ (Figure 3). With increasing $\phi,\langle d\rangle$ starts at around $20 \mu \mathrm{m}$ and then increases before it saturates at around $60 \mu \mathrm{m}$ for $\phi=70 \%$. This linear increase of $\langle d\rangle$ is possibly related to the increase in the bending modulus of the lamellar stack, whose lamellar spacing decreases with increasing $\phi$. The width $\langle d\rangle \approx 60 \mu \mathrm{m}$ remains constant for $\phi \gtrsim 80 \%$, where inverted micelles $\left(\mathrm{L}_{2}\right)$ are present in the initial droplet. This is consistent with the $\mathrm{L}_{2}$ phase first transforming into the most concentrated $\mathrm{L}_{\alpha}$ phase, and myelins then growing out of this concentrated $\mathrm{L}_{\alpha}$ phase. Moreover, the myelins have a relatively low polydispersity (indicated by the bars in Figure 3). The distribution $N(d)$ was monitored for up to $2 \mathrm{~h}$ and was found to be independent of time. Furthermore, the myelins are stable for hours without noticeable changes in their morphology.

The average myelin width $\langle d\rangle$ also depends on the sample thickness $b$ (Figure 4). For sample thicknesses $b<20 \mu \mathrm{m}$, the projected average width is $\langle d\rangle \approx 20 \mu \mathrm{m}$ independent of $b$, and hence the myelins must be flattened cylinders in this range. These myelins are also relatively unstable; within minutes they dissolve behind the growing tips. With increasing thickness, $20 \mu \mathrm{m}<b<$ $600 \mu \mathrm{m},\langle d\rangle$ also increases, with the increase in $\langle d\rangle$ being steeper for larger $\phi$. Interestingly, in this range, the average myelin width $\langle d\rangle$ depends on the sample thickness $b$ but is considerably smaller than $b$. Within this range we do not expect any direct influence of the confining walls on the mechanism for myelin formation, nor do we find any evidence for this. We cannot however rule out such an effect in the regime where flattened cylinders form, that is, 


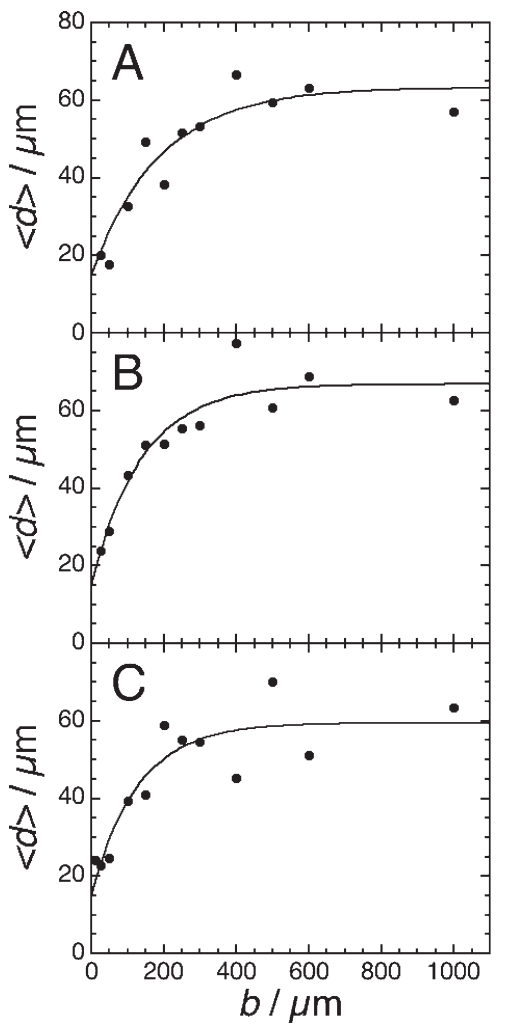

Figure 4. Average myelin width $\langle d\rangle$ as a function of sample thickness $b$ for different initial surfactant mass fractions $\phi$ of (A) $55 \%$, (B) $65 \%$, and (C) $75 \%$. The lines are guides to the eye.

$b<20 \mu \mathrm{m}$. A maximum width $\langle d\rangle \approx 60 \mu \mathrm{m}$ is reached for large sample thicknesses $b>600 \mu \mathrm{m}$. For large $(b>600 \mu \mathrm{m})$ as well as small $(b<20 \mu \mathrm{m})$ sample thicknesses $b,\langle d\rangle$ does not significantly depend on $\phi$ so that, independent of the conditions, the myelin width ranges between $20 \mu \mathrm{m} \lesssim\langle d\rangle \lesssim 60 \mu \mathrm{m}$. The main difference is that the increase in $\langle d\rangle$ occurs at smaller $b$ for larger $\phi$. Upon increasing $b$ the morphology also changes. It evolves from a tight network with a large fraction of branched myelins to a looser network of rather curved myelins (Figure 1).

3.2. Myelin Growth Kinetics. For a quantitative investigation of the myelin growth kinetics we used a home-built microscope with a variable magnification between 0.5 and 2.5. ${ }^{43,44}$ This allows us to (just) resolve individual myelins and, at the same time, to follow the growth of many myelins. It thus provides significantly improved statistics. With this apparatus we follow the formation and growth of myelins (Figure 5). Because of their structure, the myelins appear as a dark rim. The myelins grow from the edge of the initial droplet into the bulk water. Although the concentration within the droplet must decrease, we observe no significant change here, in contrast to observations in the lecithin system.

Our apparatus, as with any conventional optical microscope, provides a projection of the sample. This allows us to determine the area of the droplet (including the myelins) $A(t)=\pi R^{2}(t)$ with an average radius $R(t)$. From this and the average radius of the initial droplet $R\left(t_{0}\right)=R_{0}$, the average myelin length $L(t)=$ $R(t)-R_{0}$ can be deduced (Figure 6). Because of the projection, the length $L(t)$ corresponds to the longest myelins (section 3.3). In agreement with previous studies, ${ }^{1,2}$ the myelin length $L(t)$ initially shows a square-root dependence on time

$$
L(t)=\left(S\left(t-t^{\prime}\right)\right)^{1 / 2}
$$

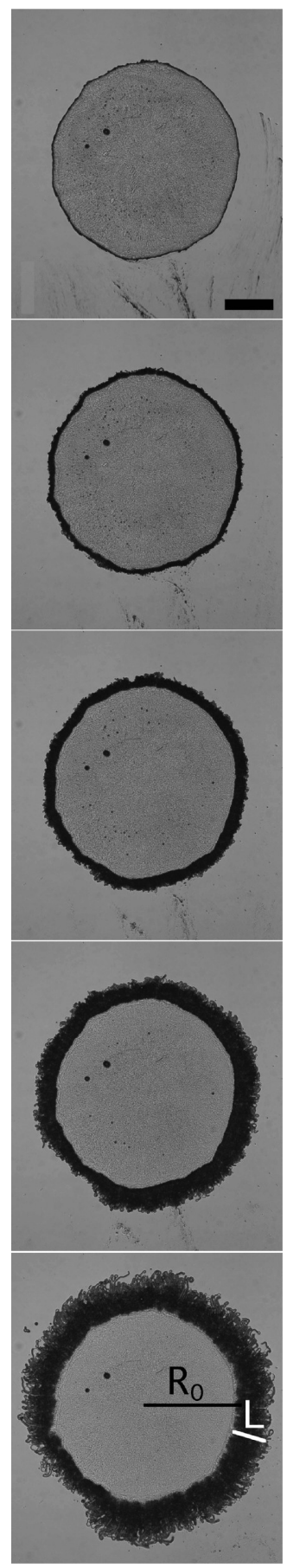

Figure 5. Myelin growth after a droplet of lamellar phase has been contacted with water. The images are taken at different times after contact; $t=1.2 \mathrm{~s}, 2.4 \mathrm{~min}, 23 \mathrm{~min}, 2.1 \mathrm{~h}$, and $9.7 \mathrm{~h}$ (top to bottom). The initial surfactant mass fraction was $\phi=70 \%$, and the sample thickness $b=200 \mu \mathrm{m}$. The initial average radius $R_{0}$ and the average myelin length $L$ are indicated. The scale bar corresponds to $2 \mathrm{~mm}$.

with the swelling parameter $S$ and a relatively short delay time $t^{\prime}$ which takes into account the delay in the onset of swelling and myelin growth. At later times the observed growth is slower, subdiffusive, due to changes in the lamellar phase caused by its swelling. These changes include the increased lamellar spacing, which increases the water mobility but also reduces the osmotic driving (see below) as well as the structure and frequency of defects. At very long times also the effect of the circular geometry is expected to become important. 


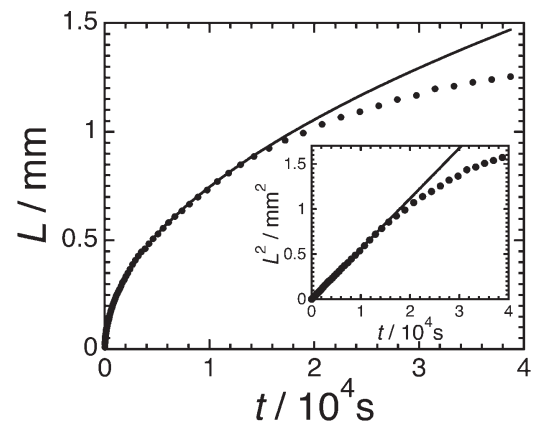

Figure 6. Time dependence of the average myelin length $L(t)$ for an initial surfactant mass fraction $\phi=65 \%$ and a sample thickness $b=200 \mu \mathrm{m}$. The line represents a fit of $L(t) \sim\left(t-t^{\prime}\right)^{1 / 2}$ to the data at early times with $t^{\prime}$ a very small delay time. The inset contains the same data, but in another representation, $L^{2}(t)$ as a function of $t$, such that the fit is a straight line.

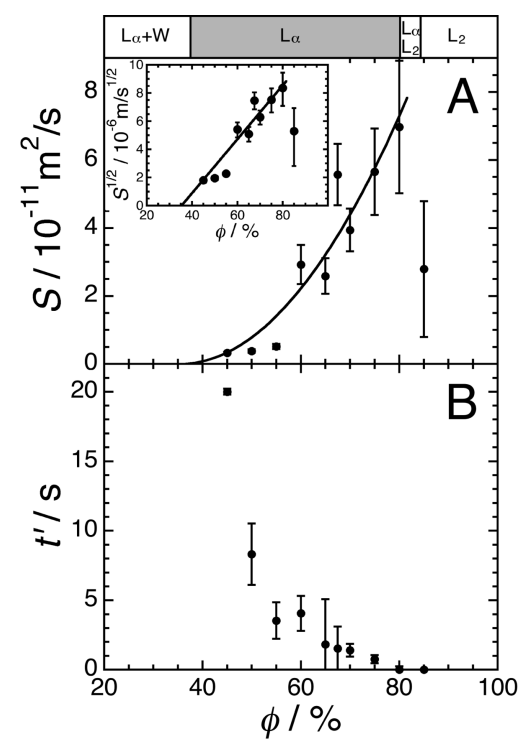

Figure 7. (A) Swelling parameter $S$ and (B) delay time $t^{\prime}$ as a function of the initial surfactant mass fraction $\phi$ for a sample thickness $b=200 \mu \mathrm{m}$. The inset contains the same data, but in another representation, $S^{1 / 2}(\phi)$ as a function of $\phi$, such that the fit is a straight line. Five individual measurements for each composition have been averaged. A schematic phase diagram for corresponding $\phi$ and temperature is shown above.

The dependence of the swelling parameter $S$ on the initial surfactant mass fraction $\phi$ has been investigated for a fixed sample thickness $b=200 \mu \mathrm{m}$, where stable myelins are formed (Figure 7A). The swelling parameter $S$ is very small for $\phi$ close to the coexistence of $L_{\alpha}$ and water, i.e., for an already almost maximally swollen lamellar phase. With increasing $\phi, S$ increases to reach $S \approx 7 \times 10^{-11} \mathrm{~m}^{2} \mathrm{~s}^{-1}$. For $\phi>80 \%$ and thus with an initial $\mathrm{L}_{2}$ phase, $S$ decreases again. The delay time $t^{\prime}$ decreases with increasing $\phi$ (Figure 7B), but even for small $\phi$ it still only reaches values $\left(t^{\prime} \approx 20 \mathrm{~s}\right)$ small compared to the time scale of myelin growth (Figure 6).

The magnitude of the swelling parameter $S$ is similar to values determined earlier: for the same system $\left(\mathrm{C}_{12} \mathrm{E}_{3}\right)$, pure surfactant ( $\mathrm{L}_{2}$ phase) was contacted with water in a cell of height $b=45 \mu \mathrm{m} .{ }^{1}$ After a few seconds $\left(t^{\prime}=9.4 \mathrm{~s}\right)$ myelins started to grow and the swelling parameter was determined to be $S=(8.5 \pm 0.5) \times$ $10^{-11} \mathrm{~m}^{2} \mathrm{~s}^{-1}$. In another nonionic system $\left(\mathrm{C}_{12} \mathrm{E}_{6}\right), S=21.9 \times$ $10^{-11} \mathrm{~m}^{2} \mathrm{~s}^{-1}$ was found. ${ }^{16}$ Also in lecithin systems the growth has been followed quantitatively, yielding $S=10 \times 10^{-11} \mathrm{~m}^{2} \mathrm{~s}^{-1}$ for egg yolk phosphatidylcholine. ${ }^{22}$ It is reassuring that these values for $S$ are comparable to the values reported here.

The dependence of $S$ on $\phi$ is consistent with the osmotic pressure, $\Pi$, being the driving force for the swelling of lamellar phase and hence myelin growth. ${ }^{14-16,25}$ With increasing $\phi$ and thus decreasing water content of the surfactant droplet, the concentration difference, and hence osmotic pressure difference between the surfactant phase and bulk water, increases. An increase in osmotic pressure results in a larger driving force for swelling. This is consistent with the observed increase in $S(\phi)$ and also with the decrease in the delay time $t^{\prime}(\phi)$ with $\phi$, i.e., the time needed to form myelins and establish a myelin growth with a $t^{1 / 2}$ dependence (Figure 7).

We now estimate the swelling parameter $S$ based on this model. $S$ is proportional to a concentration-dependent prefactor $\gamma$ and the interdiffusion coefficient $D$, itself determined by the mobility of water in the surfactant phase, $\xi_{0}$, and a thermodynamic term, essentially the osmotic compressibility of the surfactant phase $(\partial \Pi / \partial \phi)^{-1}$ :

$$
S=\gamma D=\gamma(1-\phi)^{2} \xi \frac{\partial \Pi}{\partial \phi}
$$

The prefactor is given by $\gamma=(4 / \pi)\left(\left(\phi-\phi_{\mathrm{c}}\right) / \phi_{\text {cyl }}\right)^{2}$ and takes into account both the difference $\left(\phi-\phi_{c}\right)$ of the volume fraction $\phi$ to the surfactant volume fraction at the $\mathrm{L}_{\alpha}$ to $\mathrm{L}_{\alpha}+\mathrm{W}$ boundary, $\phi_{\mathrm{c}} \approx 37 \%,{ }^{30}$ which drives the swelling, and the close-packed volume fraction of the densely packed myelins, $\phi_{\text {cyl }}=90.7 \%$. ${ }^{1}$ The mobility $\xi=(2 / 3) \xi_{0}=(2 / 3) d_{\mathrm{w}}{ }^{2} /(12 \eta)^{45}$ depends on the viscosity $\eta$ and the thickness of the water layer $d_{\mathrm{w}}=d_{\mathrm{b}}(1-\phi) / \phi$ with $d_{\mathrm{b}}$ being the bilayer thickness. The factor $2 / 3$ takes into account the possible orientations of the lamellar phase with respect to the interface and that flow through a bilayer is negligible compared to flow along a bilayer. The osmotic pressure, $\Pi$, of a lamellar phase formed by a (dilute) nonionic surfactant is dominated by undulations and is given by ${ }^{46}$

$$
\Pi=\frac{3 \pi^{2}}{64} \frac{(k T)^{2}}{\kappa} \frac{1}{d_{\mathrm{b}}{ }^{3}}\left(\frac{\phi}{1-\phi}\right)^{3}
$$

where $\kappa$ and $k$ are the bending modulus and Boltzmann's constant, respectively. We thus obtain for the swelling parameter

$$
S(\phi)=\frac{\pi(k T)^{2}}{32 \phi_{\mathrm{cyl}}{ }^{2} \eta d_{\mathrm{b}} \kappa}\left(\phi-\phi_{\mathrm{c}}\right)^{2}=\chi\left(\phi-\phi_{\mathrm{c}}\right)^{2}
$$

On the basis of literature values for the bilayer thickness $d_{\mathrm{b}}=$ $2.5 \mathrm{~nm},{ }^{39-41}$ bending modulus $\kappa=2.5 k T$ (taken from a similar system, $\left.\mathrm{C}_{12} \mathrm{E}_{6}{ }^{46}\right)$ and viscosity of water $\eta=1.0 \times 10^{-3} \mathrm{~Pa} \mathrm{~s}^{47}$ we expect $\chi^{\text {calc }}=8 \times 10^{-11} \mathrm{~m}^{2} / \mathrm{s}$. A fit of the $\left(\phi-\phi_{\mathrm{c}}\right)^{2}$ dependence to the data in the lamellar region (Figure $7 \mathrm{~A}$, solid line) results in good agreement with the data. The fit yields $\phi_{\mathrm{c}}{ }^{\mathrm{fit}}=35 \pm 6 \%$ consistent with the observed phase boundary and $\chi^{\text {fit }}=(36 \pm 12) \times$ $10^{-11} \mathrm{~m}^{2} / \mathrm{s}$, similar to the predicted value $\chi^{\text {calc }}$. This is encouraging since $\chi^{\text {calc }}$ is based on a very simple model and involves no free parameters. The model neglects, for example, that the water mobility $\xi$ is affected by bilayer undulations, the extent and hydration of the ethylene glycol headgroups, the polydispersity in the lamellar spacing, and defects in the lamellar structure.

(45) Brochard, F.; de Gennes, P. G. Pramana Suppl. 1975, 1, 1-21.

(46) Bagger-Jörgensen, H.; Olsson, U. Langmuir 1996, 12, 4057-4059.

(47) Raviv, U.; Laurat, P.; Klein, J. Nature 2001, 413, 51-54. 


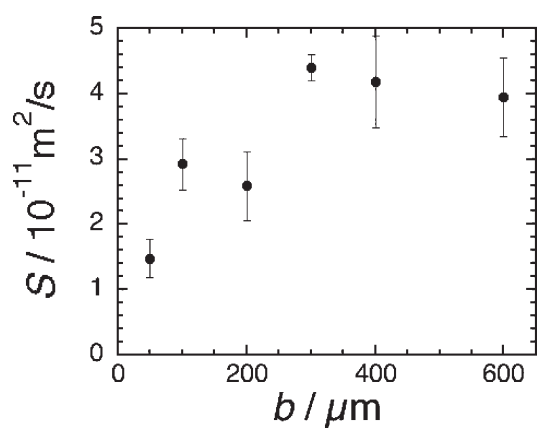

Figure 8. Experimentally determined swelling parameter $S$ as a function of sample thickness $b$. The initial surfactant mass fraction was $\phi=65 \%$.

We also investigated the dependence of the swelling parameter $S$ on the sample thickness $b$ (Figure 8 ). With increasing $b$, the observed $S$ increases and saturates for $b>300 \mu \mathrm{m}$. As will be shown in the following section, this dependence is due to the creaming of myelins which results in a slanted rather than a vertical interface (Figures 9 and 10).

3.3. Height Dependence of Myelin Growth. Conventional microscopy only provides a projection onto the $x y$-plane. We thus complemented the experiments by confocal microscopy to obtain 3-D information on the myelin growth. Consistent with the observations by conventional microscopy, the myelin/water interface progresses into the bulk water (Figure 9). Confocal microscopy furthermore reveals how the interface evolves in the $z$-direction. The myelins grow from the initially vertical interface. While growing, the myelins cream to the top of the sample as, due to the circular geometry of the droplet (or any local curvature of the interface), more space becomes available at the top of the sample. The creaming is driven by the lower density of myelins compared to the density of the surrounding water, which results from the density difference between surfactant $\left(\rho_{\mathrm{C}_{12} \mathrm{E}_{3}}\left(20^{\circ} \mathrm{C}\right)=\right.$ $\left.0.927 \mathrm{~g} / \mathrm{cm}^{3}\right)$ and water $\left(\left(\rho_{\mathrm{H}_{2} \mathrm{O}}\left(20{ }^{\circ} \mathrm{C}\right)=0.998 \mathrm{~g} / \mathrm{cm}^{3}\right)\right.$. The initially vertical interface thus becomes progressively slanted during myelin growth. This slant is particularly evident in individual slices from a $z$-scan, which show the myelins, especially their tips, as bright features (Figure 10, with the surfactant solution located to the left and the water to the right). The area representing the surfactant droplet and the myelins (left of image) decreases on moving toward the bottom of the sample (Figure 10, top to bottom).

From the individual slices, the positions of the myelin tips in the horizontal $x y$-planes were quantitatively determined as a function of the vertical $z$-position and time $t$ since contact; the $z$-profile $\mathrm{q} L(z, t)$ (Figure 11). The sample was scanned upward as well as downward and the results averaged. This reduces artifacts introduced by the finite scan speed, since the growth is approximately linear on the time scale needed to scan in both directions (about $4 \mathrm{~min}$ ). At early times the nearly vertical interface compromises the $z$-resolution due to scattering within the surfactant phase, but for longer times the slope of the interface allows for a $z$-resolution $\Delta z=5 \mu \mathrm{m}$. The absolute position of the profiles, i.e., the distance of the myelin tips (the $z$-profile) from the initial $\mathrm{L}_{\alpha} /$ water interface, that is the myelin length $L$, cannot be deduced from the confocal microscopy experiments because the sample was moved between the determination of individual $z$-profiles. Moreover, the field of view is small; comparable to conventional microscopy (Figure 1) but very considerably smaller than with our home-built microscope (Figure 5). The profiles are thus based on a limited number of myelins in a restricted area, which leads to poor
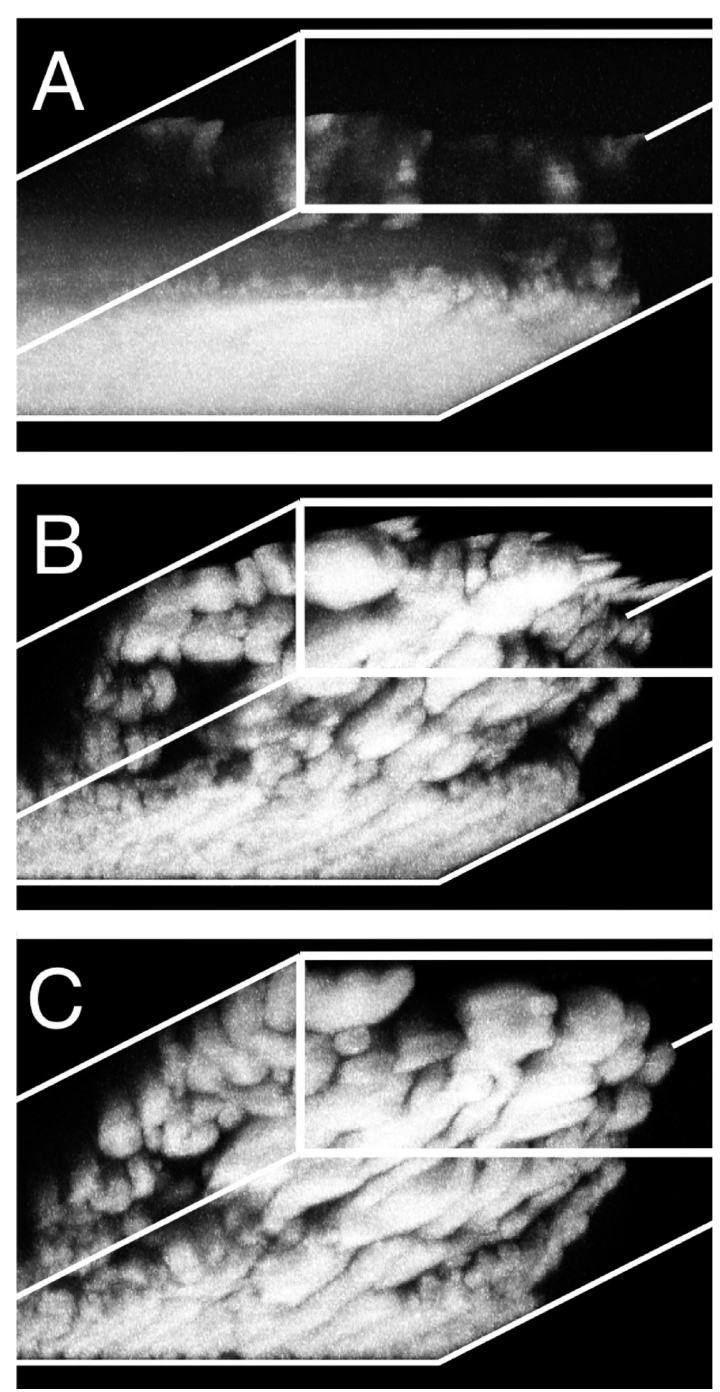

Figure 9. Myelin growth after a droplet of lamellar phase has been contacted with water as observed by confocal microscopy. The myelins grow to the right toward the observer and float to the top of the sample cell. The images are taken at different times after contact; (A) $t=5 \mathrm{~min}$, (B) $16 \mathrm{~min}$, and (C) $27 \mathrm{~min}$. The initial surfactant mass fraction was $\phi=65 \%$, and the sample thickness $b=300 \mu \mathrm{m}$. The white frame represents the field of view.

statistics and can result in profiles which are not representative of the entire sample. Nevertheless, the qualitative features and the trends in the profiles are believed to be reliable.

The profiles $L(z, t)$ show that the myelin/water interface moves into the bulk water (Figure 11A), consistent with the results from conventional microscopy. They quantify the observation that myelin growth progresses faster at the top of the sample, which leads to an increasing slant of the interface. Moreover, a region with a roughly vertical interface develops at the top of the sample, whose thickness corresponds, at later times of our observations, to the myelin width $\langle d(b)\rangle$ (gray areas in Figure 11). This is consistent with the results from microscopy, indicating a cylindrical myelin shape for the range $100 \mu \mathrm{m}<b<400 \mu \mathrm{m}$. The cylindrical shape seems to be more stable than the noncylindrical shape found at $b<20 \mu \mathrm{m}$ (section 3.1). ${ }^{4,5}$

The confocal microscopy images furthermore indicate that myelin formation is independent of the vertical position $z$; the same amount of swollen lamellar phase leaves the droplet at all heights $z$. This seems in contradiction to the observed dependence of the swelling parameter $S$ on sample thickness $b$ (Figure 8). 

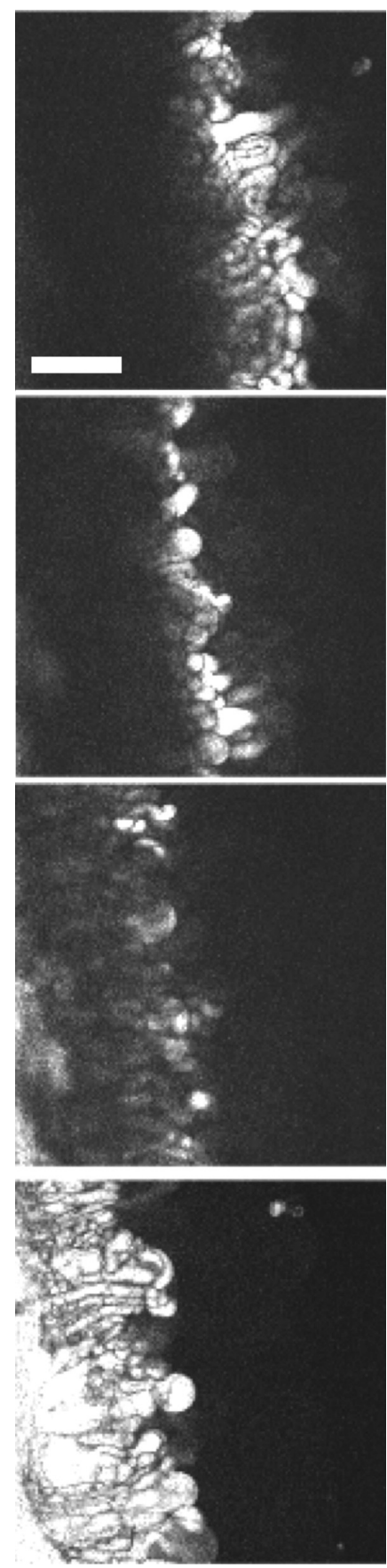

Figure 10. Slices from a confocal microscopy scan at different depths below the top glass plate; $z=-80 \mu \mathrm{m},-160 \mu \mathrm{m},-240 \mu \mathrm{m}$, and $-320 \mu \mathrm{m}$ (top to bottom). The bright features are the myelins, especially their tips. In the slices the surfactant solution is located to the left and the water to the right of the myelins. The initial surfactant mass fraction was $\phi=65 \%$, the total sample thickness $b=400 \mu \mathrm{m}$, and the time since contact $t=25 \mathrm{~min}$. The scale bar represents $200 \mu \mathrm{m}$ with a field of view of $821 \times 821 \mu \mathrm{m}^{2}$.

However, using our home-built microscope, the swelling parameter $S$ was determined from a projection onto the $x y$-plane and is thus based on the fastest growing myelins with length $L_{\max }$ at the top of the sample instead of an average length $\langle L\rangle$ of the entire $z$-profile. This average length $\langle L\rangle$ should be used to calculate an average swelling parameter $\langle S\rangle=\left(\langle L\rangle / L_{\max }\right)^{2} S$. The ratio between the average, $\langle L\rangle$, and maximum, $L_{\max }$, lengths of the myelins is, for small sample thicknesses $b$, expected to be close to one since creaming is not significant (or is impossible) in this case
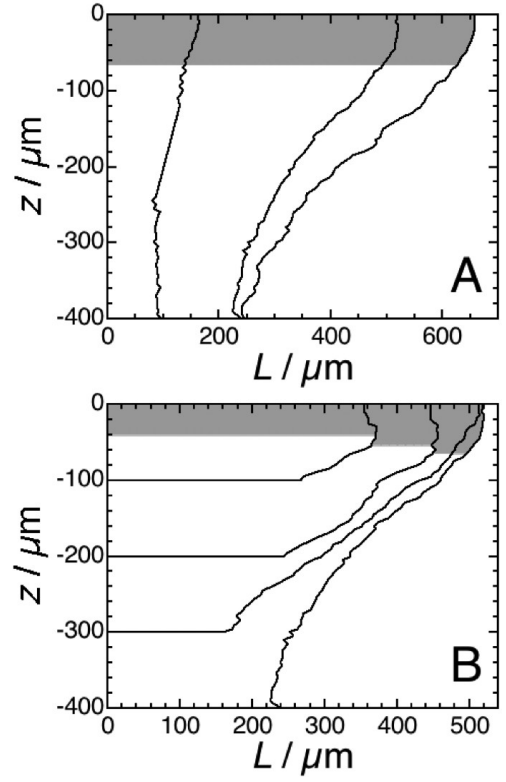

Figure 11. Myelin length $L(z, t)$ in a height $z$ as determined by confocal microscopy $(z=0 \mu \mathrm{m}$ is defined to be at the top of the sample). The $z$-profiles are shown for (A) different times $t$ after contact (left to right: $5 \mathrm{~min}, 16 \mathrm{~min}$, and $25 \mathrm{~min}$; all for $b=400 \mu \mathrm{m}$ ) and (B) different sample thicknesses $b$ (left to right: $100 \mu \mathrm{m}$, $200 \mu \mathrm{m}, 300 \mu \mathrm{m}$, and $400 \mu \mathrm{m}$; at about similar times $t=15 \mathrm{~min}$, $18 \mathrm{~min}, 16 \mathrm{~min}$, and $16 \mathrm{~min}$, respectively). The initial surfactant mass fraction was $\phi=65 \%$. The profiles have been shifted in $L$-direction (along the myelins) by an arbitrary amount because the absolute position of the profiles could not be determined. The gray areas in (A) and (B) indicate the myelin width $\langle d(b)\rangle$ for the corresponding sample thickness $b$.

and the interface is thus vertical. With increasing sample thickness $b$, the ratio $\langle L\rangle / L_{\max }$ decreases and approaches about 0.5 for large sample thicknesses $b$ since the profile is about linear (Figure 11). We thus expect that the observed swelling parameter $S$ is close to the average swelling parameter $\langle S\rangle$ for small sample thicknesses $b$ and then increases to about $S=4\langle S\rangle$ for large $b$. This is indeed observed (Figure 8). This indicates that the dependence of $S$ on sample thickness $b$ is due to creaming which causes a slant of the interface. The observed two-dimensional projection of the myelin growth can thus be rationalized in the context of the threedimensional myelin growth characteristics; the observed apparent dependence of myelin growth on sample thickness is caused by the projection of a slanted interface. A quantitative description is currently precluded, since this requires more complete and more precise three-dimensional data to be available. Nevertheless, our results indicate that myelin formation occurs homogeneously over the whole interface. Furthermore, water flux into the lamellar phase seems not significantly affected by the presence of (different amounts of) myelins and is thus also independent of height $z$.

\section{Conclusion}

We have studied the morphology and growth of myelins in aqueous surfactant solutions containing the nonionic surfactant $\mathrm{C}_{12} \mathrm{E}_{3}$. These solutions were investigated using imaging techniques which provide a projection of the sample, namely optical microscopy with different magnifications, and a complete 3-D reconstruction using confocal microscopy. Combining these techniques, we obtained detailed information on the three-dimensional shape and growth of myelins. We determined their width, length, 
and depth profile as a function of time, thus obtaining quantitative information on their growth behavior.

Upon contact with water, myelins grow from the interface of the surfactant solution into the water. In general, the myelins are stable and have a cylindrical shape. However, if the sample is very thin with a thickness $b<20 \mu \mathrm{m}$, noncylindrical myelins form which tend to be unstable. The width of the myelins was modeled with a Schultz distribution. The average width $\langle d\rangle$ ranges between about 20 and $60 \mu \mathrm{m}$ and increases with the initial surfactant mass fraction $\phi$ and thickness of the sample $b$. For all conditions a relatively small polydispersity was found.

Myelin growth starts after some relatively short delay time $\left(t^{\prime}<20 \mathrm{~s}\right)$, which decreases with increasing initial surfactant mass fraction $\phi$. During the first few hours, the initial growth follows a diffusion-like behavior; i.e., the length $L(t)$ increases with a square-root dependence $L(t) \sim t^{1 / 2}$, and at later times, the growth is slower. We focused on the initial, diffusion-like growth and determined the growth rate, characterized by the swelling parameter $S$, and found it to depend on the initial surfactant mass fraction $\phi$ and, seemingly, the sample thickness $b$.

The dependence of the observed swelling parameter $S$ on sample thickness $b$ can be attributed to a slanted rather than a vertical interface between the myelins and the water, which has been revealed by confocal microscopy. The reason for this slanted interface is a density difference between myelins and water which leads to creaming and thus faster progression of the myelin front at the top of the sample. This maximum length $L_{\max }(t)$ is observed in a projection as obtained by optical microscopy and is used to determine $S$. In contrast, an average length $\langle L(t)\rangle$ is more representative of myelin growth and should be used instead. An estimate indicates that the corresponding average swelling parameter $\langle S\rangle$ does indeed not depend on sample thickness $b$. This implies that myelin formation is independent of both sample thickness $b$ and position at the interface and that the water flux is not significantly hindered by the myelins.

The initial surfactant mass fraction $\phi$ was found to affect the swelling parameter $S$. Using the interdiffusion coefficient, we related $S$ to the mobility of water in the surfactant phase and the osmotic pressure difference between the lamellar phase and water. ${ }^{25}$ Since the dependence of the mobility and the osmotic pressure on the properties of the lamellar phase are known, ${ }^{45,46}$ the dependence of $S$ on $\phi$ can be calculated; $S(\phi) \sim\left(\phi-\phi_{\mathrm{c}}\right)^{2}$. This dependence was found to agree with our experimental observations and also the magnitude of $S$ is of the order of our experimental values. This indicates that myelin growth is indeed driven by the osmotic pressure difference and limited by the mobility of water within the surfactant phase.

Acknowledgment. L.R. thanks R. Stolarski and M. KicińskaHabior (Warsaw University) for making her initial visit to the University of Edinburgh possible. We thank P. Clegg, H. Diamant, R. G. Laughlin, and A. Schofield for very helpful discussions. The School of Chemistry is part of the EaStCHEM joint Chemistry Research School. We acknowledge the financial support of the Scottish Funding Council. Confocal microscopy was performed in the Collaborative Optical Spectroscopy Micromanipulation \& Imaging Center (COSMIC) at the University of Edinburgh. This work was funded in part by EPSRC EP/E030173. L.R. acknowledges financial support from the Marie Curie Early Stage Training Network on "Biomimetic Systems" (Contract MEST-CT-2004-504465). M.E.C. holds a Royal Society Research Professorship. 